\title{
Formas de perdas de cobre e fósforo em água de escoamento superficial e percolação em solo sob aplicações sucessivas de dejeto líquido de suínos
}

\author{
Forms of losses of copper and phosphorus in water of runoff and percolation in soil with \\ successive pig slurry application
}

\author{
Eduardo Girotto ${ }^{\mathrm{I}}$ Carlos Alberto Ceretta $^{\mathrm{I}^{*}}$ Danilo Rheinheimer dos Santos ${ }^{\mathrm{I}}$ Gustavo Brunetto $^{\mathrm{II}}$ \\ Juliano Gomes de Andrade ${ }^{\text {III }}$ Jovani Zalamena ${ }^{\text {IV }}$
}

\section{RESUMO}

As aplicações sucessivas de dejeto líquido de suínos podem aumentar os teores de cobre e fósforo na camada superficial do solo, aumentando as perdas de suas formas por escoamento superficial e percolação. $O$ trabalho teve por objetivo avaliar as perdas e as formas de cobre e de fósforo na solução escoada, na superfície e percolada em solo submetido a aplicações sucessivas de dejeto líquido de suínos, em sistema plantio direto. $O$ experimento utilizado neste estudo foi instalado no ano de 2000, sobre um solo Argissolo Vermelho distrófico arênico. Os tratamentos foram aplicações sucessivas de 0, 40 e $80 \mathrm{~m}^{3} \mathrm{ha}^{-1}$ de dejeto líquido de suínos a lanço e na superfície do solo, em sistema plantio direto. Para este trabalho, foram coletadas soluções escoadas e percoladas, durante o ciclo da aveia preta (Avena strigosa), no inverno de 2006, quando o solo apresentava um histórico de 16 aplicações de dejeto. Nas soluções, foi quantificado o sedimento e determinados os teores de $\mathrm{Cu}, \mathrm{P}$, cátions e ânions, $\mathrm{pH}$, carbono orgânico dissolvido (COD) e condutividade elétrica (CE). Usando o programa Visual MINTEQ, versão 2.15, se realizou a especiação iônica de Cu e P nas soluções. As maiores perdas de cobre e fósforo do solo ocorrem por escoamento superficial, em áreas submetidas a aplicações sucessivas de dejeto líquido de suínos em sistema plantio direto. O potencial contaminante desses elementos ficou evidenciado pela presença do cobre na forma predominante orgânica $(\mathrm{CuCOD})$ e do fósforo nas formas livres $\left(\mathrm{HPO}_{4}^{2-}\right.$ e $\left.\mathrm{H}_{2} \mathrm{PO}_{4}^{-}\right)$, na solução escoada.

Palavras-chave: adubação orgânica, poluição do solo, contaminação ambiental.

\section{ABSTRACT}

Successive pig slurry application can increase the copper and phosphorus levels in the soil and the losses of these elements by surface runoff and percolation. The objective of this study is to evaluate the losses and the forms of both copper and phosphorus in the surface runoff and also in/on the percolation solution, on soils with successive pig slurry application under no tillage system. The experiment was installed in 2000, at Typic Hapludalf soil. The treatments consisted of successive applications of 0,40 and $80 \mathrm{~m}^{3} \mathrm{ha}^{-1}$ of pig slurry applied on soil surface in no tillage system. Samples of surface runoff and percolated solutions were collected during the life cycle of oat (Avena strigosa) in the 2006 winter, when the soil had a history of 16 pig slurry applications. The following parameters have been evaluated: $\mathrm{Cu}, \mathrm{P}$, cation, anion and $\mathrm{pH}$, dissolved organic carbon and electric conductivity. The Visual MINTEQ program version 2.15 was utilized to estimate the copper and phosphorus ionic speciation. The surface runoff is the most important form of copper and phosphorus loss of the soil. In addition, copper was found in the organic form ( $\mathrm{CuCOD)}$ and phosphorus in the free forms $\left(\mathrm{HPO}_{4}^{2-} \mathrm{e}_{2} \mathrm{PO}_{4}^{-}\right)$in the surface runoff solution. Thus, these elements could be an important source of water contamination in places where pig farming is widespread.

Key words: organic fertilizer, soil pollution, environmental contamination.

'Departamento de Solos, Centro de Ciências Rurais (CCR), Universidade Federal de Santa Maria (UFSM), 97105-900, Santa Maria, RS, Brasil. E-mail: carlosceretta@smail.ufsm.br. *Autor para correspondência.

"Departamento de Engenharia Rural, Centro de Ciências Agrárias, Universidade Federal de Santa Catarina (UFSC), Florianópolis, SC, Brasil.

"IIDuPont do Brasil S/A, Divisão Pioneer Sementes, Santa Rosa, RS, Brasil.

${ }^{\mathrm{I}}$ Centro de Ciências Agroveterinárias, Universidade do Estado de Santa Catarina (UDESC), Lages, SC, Brasil. 


\section{INTRODUÇÃO}

As aplicações periódicas e superficiais de altas doses de dejeto líquido de suínos, em sistemas de cultivos sem revolvimento do solo, aumentam as quantidades de nutrientes nas camadas superficiais do solo. Logo, as transferências de poluentes (microorganismos, hormônios e elementos químicos) aos mananciais de água superficiais e subsuperficiais são potencializadas. A determinação do teor total de elementos químicos tem sido utilizada como indicador para expressar o potencial contaminante da solução escoada e percolada de solos usados como meio de descarte de dejetos de animais (GESSEL et al., 2004; BASSO et al., 2005; CERETTA et al., 2005). Entretanto, a biodisponibilidade e a mobilidade de elementos químicos, como o cobre e o fósforo, são dependentes da sua concentração na solução, da natureza da associação com outras espécies iônicas solúveis e da habilidade do solo em fornecê-los para a solução. Assim, nem todas as formas do elemento são biodisponíveis e apresentam toxidez às plantas e aos animais (CANCÉS et al., 2003). Além disso, a toxicologia dos metais pesados como o $\mathrm{Cu}$ à comunidade microbiana do solo é mais bem estimada, levando-se em consideração a especiação iônica de íons em solução (CHENG et al., 2005).

A atividade e a distribuição das espécies de um elemento químico em solução podem ser estimadas com o auxílio de softwares específicos, como o Visual MINTEQ (GUSTAFSSON, 2004). Para tal, é necessária a caracterização completa da solução em estudo, especialmente, quantificando a concentração de íons e das principais moléculas orgânicas. Esse programa é comumente usado em estudos de águas naturais, como é o caso do trabalho de MEYLAN et al. (2004). Esses autores relatam que, em águas naturais, a complexação de cobre por compostos orgânicos pode ser muito próxima de $100 \%$. Dessa forma, a especiação do metal em águas naturais seria fundamental para prever uma eventual toxidez e crucial para determinação de critérios de qualidade de águas. Isso porque, em condições naturais, a presença de altas concentrações de compostos orgânicos limita a disponibilidade e a toxidez de elementos como $\mathrm{Cu}^{2+}$ (MEYLAN et al., 2003). Além disso, estudos de especiação de elementos transferidos do solo aos sistemas aquáticos são escassos na literatura de Ciência do Solo brasileira (BERTOL et al., 2007b). Assim, a utilização dessa técnica nos estudos tradicionais de perdas de nutrientes e elementos tóxicos de áreas agrícolas poderá auxiliar na compreensão da contaminação dos mananciais aquáticos. O presente trabalho teve por objetivo avaliar as perdas e as formas de cobre e fósforo em soluções escoadas, na superfície e percoladas no perfil do solo, após aplicações sucessivas de dejeto líquido de suínos em sistema plantio direto.

\section{MATERIAL E MÉTODOS}

O experimento utilizado neste estudo se localizava na área experimental da Universidade Federal de Santa Maria (UFSM), em Santa Maria, Rio Grande do Sul (RS), (2943' S; 5342' W), sobre um solo Argissolo Vermelho distrófico arênico (EMBRAPA, 2006), com declividade média de 4\%. Em março de 2000, amostrou-se o solo da camada de $0-10 \mathrm{~cm}$ de profundidade, e os resultados das análises foram: 170, $300,530 \mathrm{~g} \mathrm{~kg}^{-1}$ de argila, silte e areia, respectivamente; $16 \mathrm{~g} \mathrm{dm}^{-3}$ de matéria orgânica (MO); $\mathrm{pH}$ em água 4,7; 0,8, 2,7 e 1,1 $\mathrm{cmol}_{\mathrm{c}} \mathrm{dm}^{-3}$ de $\mathrm{Al}, \mathrm{Ca}$ e $\mathrm{Mg}$ trocáveis, respectivamente; 15,0 e $96 \mathrm{mg} \mathrm{dm}^{-3}$ de $\mathrm{P}$ e $\mathrm{K}$ disponíveis (Mehlich 1), respectivamente, e $1,2 \mathrm{mg} \mathrm{kg}^{-1} \mathrm{de} \mathrm{Cu}$, extraído com $\mathrm{HCl} 0,1 \mathrm{~mol} \mathrm{~L}^{-1}$.

O experimento foi instalado em maio de 2000 , com parcelas de $4 \times 3 \mathrm{~m}$ e delineamento de blocos ao acaso, com quatro repetições. Os tratamentos foram as doses de 0,40 e $80 \mathrm{~m}^{3} \mathrm{ha}^{-1}$ de dejeto líquido de suínos. $\mathrm{O}$ dejeto foi a única fonte de nutrientes para as culturas, sendo sempre aplicado sobre os resíduos culturais, antes da implantação de cada cultura em sucessão no sistema plantio direto. Foram realizadas 16 aplicações de dejeto, entre o mês de maio de 2000 e maio de 2006 , totalizando 640 e $1280 \mathrm{~m}^{3} \mathrm{ha}^{-1}$ de dejeto líquido de suínos, respectivamente, para as doses de 40 e $80 \mathrm{~m}^{3}$ $\mathrm{ha}^{-1}$. No total, foram adicionados ao solo 1128 e $2256 \mathrm{~kg}$ $\mathrm{ha}^{-1}$ de $\mathrm{P}$, e 31 e $62 \mathrm{~kg} \mathrm{ha}^{-1} \mathrm{de} \mathrm{Cu}$, para as doses de $40 \mathrm{e}$ $80 \mathrm{~m}^{3} \mathrm{ha}^{-1}$ de dejeto, respectivamente.

Para a coleta da solução escoada na superfície do solo, foi instalada, no centro de cada parcela, uma armação confeccionada a partir de chapas de PVC $(0,75 \times 0,50 \mathrm{~m})$, com uma calha coletora conectada a uma mangueira e esta, a um recipiente coletor, onde a solução escoada era armazenada. Em cada parcela, também foram instalados dois lisímetros $(0,40 \mathrm{x} 0,60 \mathrm{~m})$ de tensão zero a $0,60 \mathrm{~m}$ de profundidade para coleta da solução percolada no perfil do solo, confeccionados com chapa de PVC (4mm de espessura), de maneira a favorecer o fluxo de água para um recipiente com capacidade de cinco litros. Mais detalhes podem ser obtidos em BASSO (2003).

A coleta da solução escoada na superfície e percolada no perfil do solo foi realizada após o segundo evento pluvial ocorrido 20 dias após a implantação da cultura de aveia preta (Avena strigosa), cultivada no inverno de 2006, quando ocorreu precipitação 
equivalente a $35 \mathrm{~mm}$. As amostras das soluções coletadas foram filtradas em membrana de $<0,22 \mu \mathrm{m} \mathrm{e}$, em seguida, foram determinados os valores de $\mathrm{pH}$ e condutividade elétrica (CE). Logo depois, uma subamostra das soluções foi acidificada com $\mathrm{HCl} 0,1 \mathrm{~mol}$ $\mathrm{L}^{-1} \mathrm{e}$ nela determinado os teores de cátions em solução $\left(\mathrm{Ca}^{2+}, \mathrm{Mg}^{2+}, \mathrm{Cu}^{2+}, \mathrm{Zn}^{2+}, \mathrm{Mn}^{2+}, \mathrm{Fe}^{2+}, \mathrm{K}^{+} \mathrm{e} \mathrm{Na}^{+}\right)$, de acordo com metodologia proposta por TEDESCO et al. (1995). Outra subamostra não acidificada foi utilizada para a determinação dos teores de carbono orgânico dissolvido (COD) e total (COT), segundo SILVA (2001), fosfato (MURPHY \& RILEY, 1962), sulfato, cloro e nitrato (TEDESCO et al., 1995). O restante da solução (não filtrada) foi submetido à digestão ácida, segundo método 3005A(EPA, 1992), em que foram determinados os teores totais de cobre e fósforo. Além disso, uma alíquota da solução escoada foi seca em estufa com ar forçado a $60^{\circ} \mathrm{C}$ e determinada a quantidade de sedimento transferido para fora da parcela.

A especiação iônica da solução escoada e percolada foi realizada usando o programa computacional Visual MINTEQ (versão 2.15 GUSTAFSSON, 2004). Para isso, foram usadas as concentrações de cátions $\left(\mathrm{Ca}^{2+}, \mathrm{Mg}^{2+}, \mathrm{Cu}^{2+}, \mathrm{Zn}^{2+}, \mathrm{Mn}^{2+}\right.$, $\mathrm{Fe}^{2+}, \mathrm{K}^{+}$e $\left.\mathrm{Na}^{+}\right)$e de ânions $\left(\mathrm{NO}_{3}^{-}, \mathrm{PO}_{4}^{-}, \mathrm{SO}_{4}^{-}\right.$e $\left.\mathrm{Cl}^{-}\right)$, a força iônica calculada, o pH e a CE medidos na solução. As espécies ligadas aos ânions orgânicos foram estimadas com base no teor de COD, usando o modelo de "Gaussian DOM" (GRIMM et al., 1991). Foram estimadas a concentração, a distribuição percentual e a atividade das espécies de cobre e fósforo em solução.

Os resultados obtidos foram submetidos à análise de variância e, quando os efeitos foram significativos, foram ajustadas equações de regressão para cada parâmetro avaliado, com probabilidade de erro menor que $5 \%(\mathrm{P}<0,05)$.

\section{RESULTADOS E DISCUSSÃO}

Transferência de sedimentos, cobre e fósforo.

A aplicação continuada de dejeto líquido de suínos favoreceu as transferências de sedimentos por escoamento, sendo na dose de $80 \mathrm{~m}^{3} \mathrm{ha}^{-1}$ encontradas perdas de 2,7 vezes maiores do que onde não foi aplicado dejeto. Consequentemente, as quantidades de cobre e, principalmente, de fósforo transferido também aumentaram significativamente, chegando a 2,3 e 92,8 vezes maior, respectivamente, do que onde não foi aplicado dejeto (Tabela 1). Isso pode ser atribuído às aplicações sucessivas de dejetos que promovem o acúmulo de fósforo e de cobre nos primeiros centímetros do perfil do solo. Além disso, os dejetos possuem altas concentrações de sólidos, podendo provocar alteração na capacidade de infiltração de água no solo, ocasionada pelo entupimento de poros e pela formação de crosta em sua superfície, o que é perceptível logo após a aplicação do dejeto na superfície do solo.

A maior parte do cobre transferido foi na forma particulada. Na solução escoada, o cobre adsorvido às partículas perfez 86,71 e $57 \%$, enquanto que, na solução percolada, foi de 68,75 e $75 \%$ do cobre total, nas doses de 0,40 e $80 \mathrm{~m}^{3} \mathrm{ha}^{-1}$ de dejeto, respectivamente. Resultados semelhantes foram encontrados por BERTOL (2005), em que praticamente todo o cobre presente em soluções escoadas superficialmente se encontrava na forma particulada em sedimentos mais grosseiros e/ou em sedimentos

Tabela 1 - Perdas de sedimentos, cobre e fósforo por escoamento superficial e percolação no perfil do solo, em um solo com aplicações sucessivas de dejeto líquido de suínos na superfície do solo e cultivado sob sistema plantio direto.

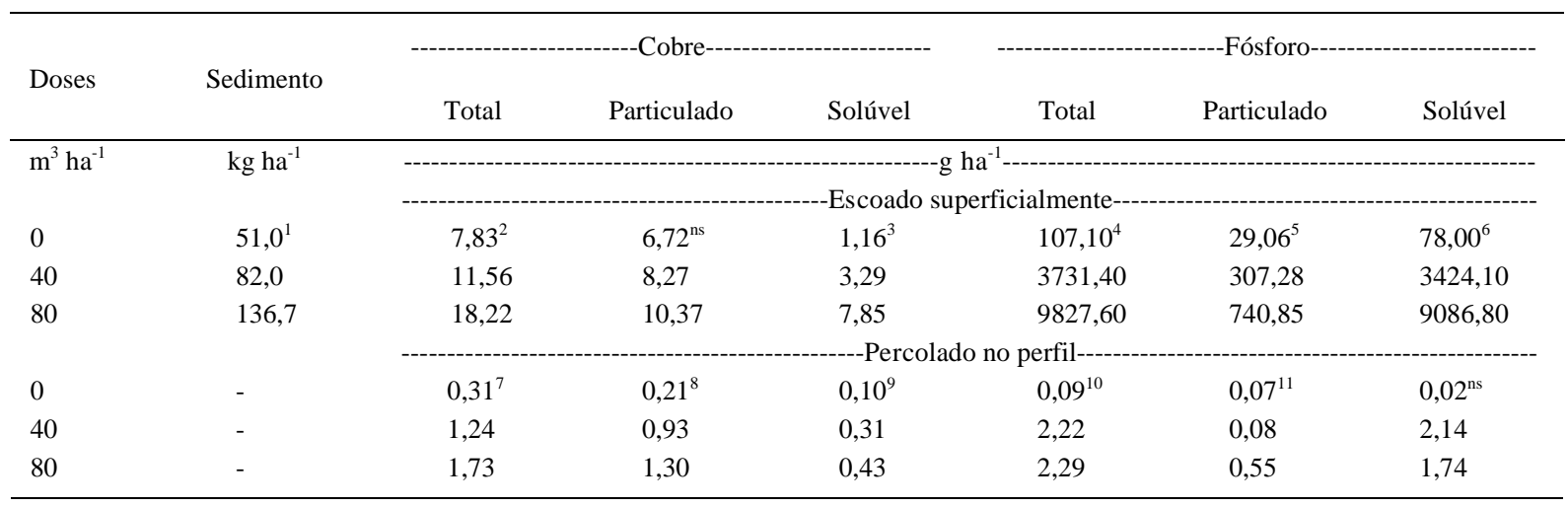

ns $=$ não significativo a 5\% de probabilidade de erro; ${ }^{(1)} \mathrm{y}=4,200+42,8520 \mathrm{x}\left(\mathrm{r}^{2}=0,97\right) ;{ }^{(2)} \mathrm{y}=7,375+0,129 \mathrm{x}\left(\mathrm{r}^{2}=0,97\right) ;{ }^{(3)} \mathrm{y}=0,755+0,084 \mathrm{x}\left(\mathrm{r}^{2}=0,96\right)$; ${ }^{(4)} \mathrm{y}=-304,88+121,51 \mathrm{x}\left(\mathrm{r}^{2}=0,98\right) ;{ }^{(5)} \mathrm{y}=3,168+8,897 \mathrm{x}\left(\mathrm{r}^{2}=0,98\right) ;{ }^{(6)} \mathrm{y}=-308,1+112,61 \mathrm{x}\left(\mathrm{r}^{2}=0,98\right),{ }^{(7)} \mathrm{y}=0,375+0,018 \mathrm{x}\left(\mathrm{r}^{2}=0,98\right) ;{ }^{(8)} \mathrm{y}=0,268+0,014 \mathrm{x}$ $\left(\mathrm{r}^{2}=0,97\right) ;{ }^{(9)} \mathrm{y}=0,118+0,004 \mathrm{x}\left(\mathrm{r}^{2}=0,96\right) ;{ }^{(10)} \mathrm{y}=0,433+0,027 \mathrm{x}\left(\mathrm{r}^{2}=0,77\right) ;{ }^{(11)} \mathrm{y}=-0,007+0,006 \mathrm{x}\left(\mathrm{r}^{2}=0,76\right)$. 
em suspensão. Contudo, mesmo que a energia de ligação do cobre com os coloides, especialmente, os orgânicos, seja alta, por causa do aumento na concentração total desse metal, a concentração do íon na forma livre tende a aumentar nas soluções escoadas superficialmente.

As quantidades de fósforo particulado transferidas nas soluções escoadas e percoladas, em relação ao total transferido, foram de $27 ; 8$ e $7 \%$ e $78 ; 4$ e $24 \%$, nas doses de 0,40 e $80 \mathrm{~m}^{3} \mathrm{ha}^{-1}$ de dejeto, respectivamente (Tabela 1 ). A predominância de formas solúveis de fósforo transferidas é decorrente da adoção do sistema plantio direto, aliado à dinâmica desse elemento no solo. Diferentemente do cobre, o fosfato não é adsorvido diretamente por coloides orgânicos, os quais são comumente encontrados nas soluções escoadas superficialmente em decorrência da aplicação de resíduos orgânicos superficiais. Desse modo, aplicações de fosfatos em doses maiores do que as exportadas do sistema (LEHMANN et al., 2005) vão paulatinamente saturando os sítios de adsorção dos coloides inorgânicos do solo, especialmente os óxidos, o que aumenta a quantidade desse nutriente na solução do solo. Na área experimental utilizada neste estudo, já foram adicionados 1128 e $2256 \mathrm{~kg} \mathrm{P} \mathrm{ha}^{-1}$, para as doses de 40 e $80 \mathrm{~m}^{3} \mathrm{ha}^{-1}$, respectivamente, o que tem proporcionado aumentos de 41 vezes acima do teor crítico agronômico, nas camadas superficiais do solo (BERWANGER et al., 2008). Aliado a isso, os compostos orgânicos dissolvidos podem competir pelos adsorventes do ânion fosfato, diminuindo a sua força de adsorção aos coloides no solo, o que aumenta a concentração na solução (HOLFORD et al., 1997). Deve ser considerado, também, que os altos valores de $\mathrm{pH}$ da solução escoada diminuem a afinidade do fosfato aos coloides em solução, pelo aumento das cargas negativas na superfície destes, o que aumenta a ocorrência de formas livres do elemento, como $\mathrm{H}_{2} \mathrm{PO}_{4}^{-} \mathrm{e}$ $\mathrm{HPO}_{4}{ }^{2-}$. Com isso, o risco de perdas de fósforo na forma solúvel, via escoamento superficial e percolação no solo, é maior, potencializando processos de eutrofização de ambientes aquáticos, como alertado por BASSO et al. (2005) e CERETTAet al. (2005).

A concentração de fósforo solúvel (fração $<0,22 \mu \mathrm{m}$ ) na solução escoada foi de 42, 286 e 600 vezes maior que a encontrada na solução percolada, para as doses de 0,40 e $80 \mathrm{~m}^{3} \mathrm{ha}^{-1}$ de dejeto, respectivamente (Tabela 2). Observa-se claramente nesses dados que a

Tabela 2 - Valores de pH, condutividade elétrica, carbono orgânico dissolvido (COD), carbono total, teores solúveis de ânions e cátions (fração $<0,22 \mu \mathrm{m}$ ) nas soluções escoadas e percoladas em um solo com aplicações sucessivas de dejeto líquido de suínos na superfície do solo e cultivado sob sistema plantio direto.

\begin{tabular}{|c|c|c|c|c|c|c|c|c|}
\hline $\begin{array}{l}\text { Doses } \\
-m^{3} h a^{-1}-\end{array}$ & $\mathrm{pH}$ & $\begin{array}{c}\mathrm{CE} \\
-\mu \mathrm{S} \mathrm{cm}^{-1}-\end{array}$ & COD & COT & $\mathrm{NO}_{3}^{-}$ & $\mathrm{Cl}$ & $\mathrm{P}$ & $S$ \\
\hline & & & ------- & ção esco & - & --o- & -0 & --.-. \\
\hline 0 & $7,0^{1}$ & $123,2^{2}$ & $9,24^{\mathrm{ns}}$ & $15,3^{3}$ & $0,28^{4}$ & $6,85^{\mathrm{ns}}$ & $0,42^{5}$ & $0,54^{\mathrm{ns}}$ \\
\hline 40 & 7,4 & 304,0 & 11,46 & 17,27 & 2,40 & 8,66 & 20,00 & 0,93 \\
\hline 80 & 7,4 & 505,0 & 12,29 & 49,09 & 3,69 & 8,99 & 39,11 & 0,67 \\
\hline 0 & $5,8^{6}$ & $191,2^{7}$ & $2,27^{8}$ & $3,23^{9}$ & $0,14^{10}$ & $5,32^{11}$ & $0,01^{12}$ & $0,13^{\text {ns }}$ \\
\hline 40 & 6,1 & 369,0 & 1,78 & 3,60 & 0,42 & 13,92 & 0,07 & 0,12 \\
\hline \multirow[t]{2}{*}{80} & 5,6 & 424,0 & 2,87 & 7,20 & 0,60 & 16,57 & 0,05 & 0,08 \\
\hline & $\mathrm{Mg}$ & $\mathrm{Ca}$ & $\mathrm{K}$ & $\mathrm{Na}$ & $\mathrm{Mn}$ & $\mathrm{Cu}$ & $\mathrm{Zn}$ & $\mathrm{Fe}$ \\
\hline 0 & $3,14^{13}$ & $4,71^{14}$ & $4,27^{15}$ & $7,63^{\mathrm{ns}}$ & $0,09^{\text {ns }}$ & $0,01^{\mathrm{ns}}$ & $0,39^{\text {ns }}$ & $1,49^{\mathrm{ns}}$ \\
\hline 40 & 25,47 & 9,16 & 14,66 & 8,36 & 0,13 & 0,02 & 0,26 & 2,72 \\
\hline 80 & 24,17 & 12,41 & 20,70 & 10,70 & 0,18 & 0,02 & 0,26 & 0,36 \\
\hline 0 & $5,37^{16}$ & $13,50^{17}$ & $1,60^{18}$ & $\begin{array}{r}\text { ão perco } \\
11,33^{19}\end{array}$ & $0,11^{\mathrm{ns}}$ & $0,01^{\mathrm{ns}}$ & $0,04^{\mathrm{ns}}$ & $0,09^{\text {ns }}$ \\
\hline 40 & 9,04 & 24,96 & 2,20 & 23,46 & 0,12 & 0,01 & 0,04 & 0,12 \\
\hline 80 & 11,12 & 22,92 & 2,20 & 29,73 & 0,17 & 0,01 & 0,08 & 0,22 \\
\hline
\end{tabular}

${ }^{\text {ns }}=$ não significativo a $5 \%$ de probabilidade de erro; ${ }^{(1)} \mathrm{y}=7,085+0,0046 \mathrm{x} \quad\left(\mathrm{r}^{2}=0,72\right) ;{ }^{(2)} \mathrm{y}=119,88+4,7719 \mathrm{x}\left(\mathrm{r}^{2}=0,99\right) ;{ }^{(3)} \mathrm{y}=0,325+0,4224 \mathrm{x}$ $\left(\mathrm{r}^{2}=0,79\right) ;{ }^{(4)} \mathrm{y}=0,418+0,0426 \mathrm{x} \quad\left(\mathrm{r}^{2}=0,98\right) ;{ }^{(5)} \mathrm{y}=0,556+0,4828 \mathrm{x} \quad\left(\mathrm{r}^{2}=0,99\right) ;{ }^{(6)} \mathrm{y}=5,985-0,0026 \mathrm{x} \quad\left(\mathrm{r}^{2}=0,17\right) ;{ }^{(7)} \mathrm{y}=211,67+2,9100 \mathrm{x} \quad\left(\mathrm{r}^{2}=0,91\right) ;{ }^{(8)}$ $\mathrm{y}=2,006+0,0075 \mathrm{x} \quad\left(\mathrm{r}^{2}=0,30\right) ; \quad(9) \quad \mathrm{y}=2,691+0,0496 \mathrm{x} \quad\left(\mathrm{r}^{2}=0,81\right) ; \quad(10) \quad \mathrm{y}=0,156+0,0058 \mathrm{x} \quad\left(\mathrm{r}^{2}=0,98\right) ; \quad(11) \mathrm{y}=6,311+0,1406 \mathrm{x} \quad\left(\mathrm{r}^{2}=0,91\right) ;$ (12) $y=0,023+0,0005 x \quad\left(r^{2}=0,42\right), \quad{ }^{(13)} y=7,078+0,2629 x \quad\left(r^{2}=0,70\right) ; \quad(14) y=4,910+0,0962 x \quad\left(r^{2}=0,99\right) ; \quad(15) y=4,995+0,2054 x \quad\left(r^{2}=0,97\right) ;$ ${ }^{(16)} \mathrm{y}=5,635+0,0719 \mathrm{x}\left(\mathrm{r}^{2}=0,97\right) ;{ }^{(17)} \mathrm{y}=15,750+0,1178 \mathrm{x}\left(\mathrm{r}^{2}=0,59\right) ;{ }^{(18)} \mathrm{y}=1,700+0,0075 \mathrm{x}\left(\mathrm{r}^{2}=0,75\right) ;{ }^{(19)} \mathrm{y}=12,307+0,2300 \mathrm{x}\left(\mathrm{r}^{2}=0,97\right)$. 
solução escoada superficialmente no solo é a principal forma de transferência de fósforo no solo. Isso reforça as recomendações agronômicas e ambientais de que as práticas de manejo de água devem ser melhoradas no sistema plantio direto (BERTOL et al., 2007a).

Os valores de $\mathrm{pH}$ da solução escoda em todos os tratamentos foram mairores que 7,0 Contudo, convérm relatar que se observa aumento significativo dos valores de $\mathrm{pH}$ da solução escoada nos tratamentos com aplicações sucessivas de 40 e $80 \mathrm{~m}^{3} \mathrm{ha}^{-1}$ de dejeto (Tabela 2). Por outro lado, na solução percolada, foram obsevados valores de $\mathrm{pH}$ menores que os encontrados na solução escoada em todos os tratamentos. Isso pode ser atribuído à reação da água com o solo, durante o seu deslocamento no perfil do solo, pois o solo deste trabalho possuía $\mathrm{pH}$ em água próximo a 5,0 nas camadas mais profundas do solo. Assim, a reação da água da chuva com a solução do solo tende a causar um processo de acidificação da solução percolada no perfil do solo. Por outro lado, a CE teve aumento significativo nas soluções escoadas superficialmente e percoladas no solo, em função do aumento das doses de dejeto (Tabela 2). O aumento da CE se deve aos aumentos significativos nas concentrações de cátions, como $\mathrm{K}^{+}$, $\mathrm{Ca}^{2+}, \mathrm{Mg}^{2+}$ e $\mathrm{Na}^{+}$, e de ânions, como $\mathrm{NO}_{3}{ }^{-}$e $\mathrm{Cl}^{-}$(Tabela 2). Além disso, os teores de COD e COT também aumentaram onde foi aplicado dejeto, com maiores valores nas soluções escoadas superficialmente (Tabela 2), devido à aplicação superficial de dejeto e ao acúmulo de matéria orgânica no solo.

Especiação do fósforo e do cobre nas soluções escoada e percolada

A maior parte do fósforo na solução percolada (fração $<0,22 \mu \mathrm{m}$ ) foi encontrada na forma monovalente $\left(\mathrm{H}_{2} \mathrm{PO}_{4}^{-}\right)$(Tabela 3 ). Por outro lado, na solução escoada, predominou a forma divalente $\left(\mathrm{HPO}_{4}^{2-}\right)$ onde foi aplicado o dejeto. O predomínio de formas divalentes ou monovalentes é explicado pelo valor do $\mathrm{pH}$ da solução, em que no valor de $\mathrm{pH}$ 7,2 há aproximadamente a mesma quantidade dessas duas formas de fósforo na solução (ZAMBROSI, 2004), como observado na solução escoada. Além disso, na solução escoada, foram detectadas quantidades consideráveis de fósforo ligado ao íon magnésio (Tabela 3), pois foram detectados altos teores desse nutriente na solução.

O cobre presente na fração $<0,22 \mu \mathrm{m}$ apresenta altos percentuais de associação ao COD (CuCOD). Na solução percolada, essa forma de cobre perfez em média $60 \%$ do cobre total solúvel, e na solução de escoamento superficial esse valor foi superior a $80 \%$ (Tabela 3 ). Além disso, semelhante ao

Tabela 3 - Distribuição das espécies de fósforo e de cobre (fração $<0,22 \mu \mathrm{m}$ ) nas soluções escoadas e percoladas em um solo com aplicações sucessivas de dejeto líquido de suínos na superfície do solo e cultivado sob sistema plantio direto.

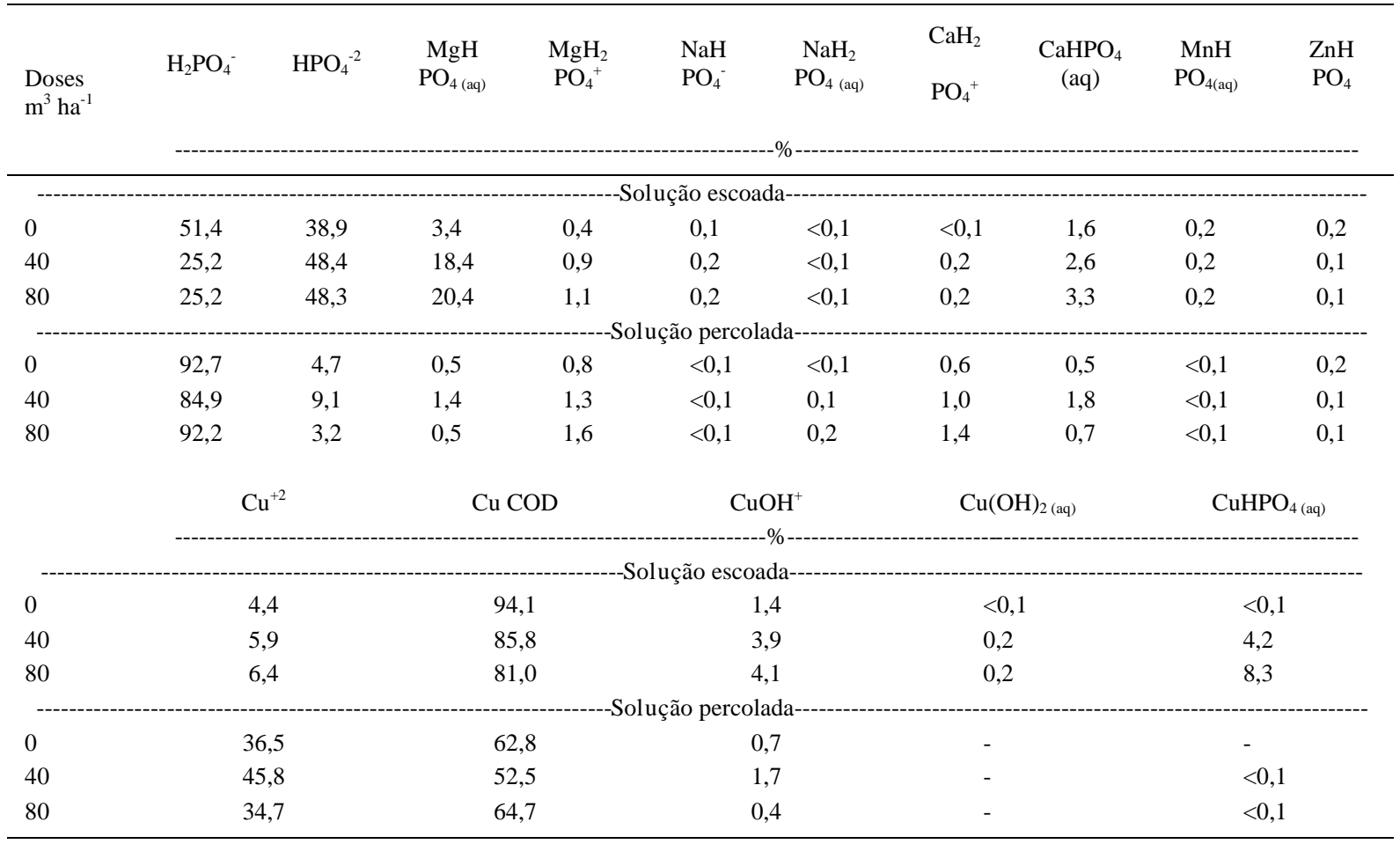


encontrado por MEYLAN et al. (2004), apenas uma pequena quantidade de cobre esteve livre ou associada ao íon fosfato. Esse comportamento se justifica pelas características do íon cobre, como alta eletronegatividade e relação carga/raio iônico, que aumentam significativamente sua afinidade pelos grupos funcionais presentes nas substâncias húmicas (McBRIDE, 1994), como observado neste estudo. Entre os grupos funcionais presentes em compostos orgânicos, os grupos carboxílicos (-COOH), fenólicos $(-\mathrm{OH})$, thióis (-SH) e aminos (- $\left.\mathrm{NH}_{2}\right)$ são os que possuem maior importância para a complexação do Cu (SMITH et al., 2002). A adsorção do cobre por substâncias orgânicas, também denominada complexação, é favorecida pelo aumento do $\mathrm{pH}$ do meio, pois favorece a dissociação dos grupos funcionais do adsorvente e o aparecimento do íon $\mathrm{Cu}^{+}$(ALDRICH et al., 2002). Esses complexos orgânicos formados são móveis no solo e podem facilitar o transporte do cobre para as águas superficiais e subsuperficiais (McCARTHY \& ZACHARA, 1989), como observado neste estudo.

\section{CONCLUSÃO}

As maiores perdas de cobre e fósforo do solo ocorrem por escoamento superficial, em áreas submetidas a aplicações sucessivas de dejeto líquido de suínos em sistema plantio direto. O potencial contaminante desses elementos ficou evidenciado pela presença do cobre na forma predominante orgânica (CuCOD) e do fósforo nas formas livres $\left(\mathrm{HPO}_{4}^{2-} \mathrm{e}\right.$ $\mathrm{H}_{2} \mathrm{PO}_{4}^{-}$), na solução escoada.

\section{REFERÊNCIAS}

ALDRICH, A.P. et al. Speciation of $\mathrm{Cu}$ and $\mathrm{Zn}$ in drainage water from agricultural soils. Environmental Science \& Technology, v.36, p.4824-4830, 2002. Disponível em: <http:/ /pubs.acs.org/doi/pdfplus/10.1021/es025813x>. Acesso em: 20 jan. 2006. doi: 10.1021/es025813x.

BASSO, C.J. et al. Dejeto líquido de suínos: II-Perdas de nitrogênio e fósforo por percolação no solo sob plantio direto. Ciência Rural, v.35, p.1305-1312, 2005. Disponível em: <http://www.scielo.br/pdf/cr/v35n6/a12v35n6.pdf>. Acesso em: 20 jan. 2007. doi: 10.1590/S0103-84782005000600012.

BASSO, C.J. Perdas de nitrogênio e fósforo com aplicação no solo de dejetos líquidos de suínos. 2003. 125f. Tese (Doutorado em Agronomia) - Universidade Federal de Santa Maria, Santa Maria, RS.

BERTOL, I. et al. Phosphorus, potassium and organic carbon concentrations in runoff water and sediments under different soil tillage systems during soybean growth. Soil \& Tillage Research, v.94, p.142-150, 2007a. Disponível em: <http:// w w w s c i e n c ed i r e t. com/s c i e n c e ? ob $=$ M Img \&_imagekey=B 6 TC $6-4$ KVXPH 6 - 1 -
$1 \& \mathrm{cdi}=5162 \&$ user $=687358 \&$ pi i=S 0167198706 $001644 \&$ orig $=$ search \& coverDate $=05 \% 2 \mathrm{~F} 31 \% 2 \mathrm{~F} 2$ $007 \&$ \& $\mathrm{k}=999059998 \& \mathrm{view}=\mathrm{c} \& \mathrm{wchp}=\mathrm{dGLbVzW}-$ zSkWb\&md5=dc 1 fd0bc725ab10ecf48b $1951685004 \mathrm{c} \& i e=/$ sdarticle.pdf>. Acesso em: 10 mar. 2008. doi:10.1016/ j.still.2006.07.008.

BERTOL, O.J. et al. Perdas de solo e água e qualidade do escoamento superficial associadas à erosão entre sulcos em área cultivada sob semeadura direta e submetida às adubações mineral e orgânica. Revista Brasileira de Ciência do Solo, v.31, p.781-792, 2007b. Disponível em: <http://www.scielo.br/pdf/ rbcs/v31n4/a18v31n4.pdf>. Acesso em: 10 mar. 2008. doi: 10.1590/S0100-06832007000400018.

BERTOL, O.J. Contaminação da água de escoamento superficial e da água percolada pelo efeito de adubação mineral e adubação orgânica em sistema de semeadura direta. 2005. 209f. Tese (Doutorado Agronomia) Universidade Federal do Paraná, Curitiba, PR.

BERWANGER, A.L. et al. Alterações no teor de fósforo no solo com aplicação de dejetos líquidos de suínos. Revista Brasileira de Ciência do Solo, v.32, p.2525-2532, 2008. Disponível em: <http://www.scielo.br/pdf/rbcs/v32n6/ v32n6a29.pdf $>$. Acesso em: 02 abr. 2009. doi: 10.1590/S010006832008000600029 .

CANCÉS, B. et al. Metal ions speciations in a soil and its solution: experimental data and model results. Geoderma, v.113, p.641355, 2003. Disponível em: <http://www.sciencedirect.com/ science? ob=MImg\&imagekey $=$ B6V67-47NF6DF-2-G\&cdi $=$ $5807 \&$ \& s e r $=687358$ \&_pi i $=$ S 00167061020 $03695 \&$ _orig $=$ search \&_coverDate $=05 \% 2$ F $31 \%$ 2F2003\&_sk $=998869996 \& v i e w=c \& w c h p=d G L b V 1 W-$ zSkzV\&md5=c51a474c7f8d5327276e5a 048468b219\&ie=/ sdarticle.pdf>. Acesso em: 20 jan. 2006. doi:10.1016/S00167061(02)00369-5.

CERETTA, C.A. et al. Dejeto líquido de suínos: I-perdas de nitrogênio e fósforo na solução escoada na superfície do solo, sob plantio direto. Ciência Rural, v.35, p.1296-1304, 2005. Disponível em: <http://www.scielo.br/pdf/cr/v35n6/ a11v35n6.pdf>. Acesso em: 20 jan. 2007. doi: 10.1590/S010384782005000600011 .

CHENG T. et al. Measurement and computation of zinc binding to natural dissolved organic matter in European surface waters. Analytica Chimica Acta, v.542, p.230-239, 2005. Disponível em: <http:// www.sciencedirect.com/science?ob=MImg\&_imagekey=B6TF4$4 \mathrm{G} 21 \mathrm{Y} 1 \mathrm{~V}-2-1 \& \mathrm{cdi}=5216 \&$ user $=687358 \& \mathrm{pii}=\mathrm{S} 000$ $3267005005088 \&$ orig $=\mathrm{search} \& \mathrm{coverDate}$ $=06 \% 2 \mathrm{~F} 29 \% 2 \mathrm{~F} 2005 \& \mathrm{sk}=994579997 \& \mathrm{view}=\mathrm{c} \& \mathrm{wchp}=\mathrm{dGLbVzW}-$ zSkzV\&md5=0bc52d914c2979f4efb9ecc4d7711bda\&ie $=/$ sdarticle.pdf $>$ Acesso em: 20 jan. 2007. doi:10.1016/ j.aca.2005.03.053.

EMBRAPA - Empresa Brasileira de Pesquisa Agropecuária Centro Nacional de Pesquisa de Solos. Sistema Brasileiro de Classificação de Solos. 2.ed. Rio de Janeiro, 2006. 374p.

EPA - Enviromnental Protection Agency. Method 3005 A. Acid digestion of waters for total recoverable or dissolved metals for analysis by flaa or icp spectroscopy. 1992. Disponível em: <http// www.epa.gov/sw-846/pdfs/3005A.pdf>. Acesso em: 20 abr. 2006. 
GESSEL, P.D. et al. Rate of fall-applied liquid swine manure: Effects on runoff transport of sediment and phosphorus. Journal of Environmental Quality, v.33, p.1839-1844, 2004. Disponível em: <http://www.prairieswine.com/database/ pdf/2066.pdf $>$. Acesso em: 20 jan. 2007.

GRIMM, D.M. et al. Continuous multiligand distribution model used to predict the stability constant of copper (II) metal complexation with humic material from fluorescence quenching data. Environmental Science and Technology, v.25, p.1427-1431, 1991. Disponível em: <http://pubs.acs.org/doi/ pdf/10.1021/es00020a010>. Acesso em: 20 jan. 2007. doi: 10.1021/es00020a010.

GUSTAFsson, J.P. Visual Minteq. Capturado em: $03 \mathrm{dez}$. 2004. Online. Disponível em: <http://www.lwr.kth.se/english/ oursoftwaer/Vminteq>.

HOLFORD, I.C.R. et al. Effects of animal effluent on the phosphorus sorption characteristics of soil. Australian Journal Soil Researsch, v.35, p.365-373, 1997. Disponível em:<http://www.publish.csiro.au/paper/S96048>. Acesso em: 20 jan. 2007. doi:10.1071/S96048.

LEHMANN, J. et al. Long-term dynamics of phosphorus forms and retention in manure-anlended soils. Environmental Science and Technology, v.39, p.6672-6680, 2005. Disponível em: <http://pubs.acs.org/doi/pdf/10.1021/ es047997g >. Acesso em: 03 mar. 2007. doi: 10.1021/ es047997g.

McBRIDE, M.B. Environmental chemistry of soils. New York, Oxford University, 1994. 406p.

McCARTHY, J.F.; ZACHARA, J.M. Subsurface transport of contaminants. Environmental Science and Technology, v.23, p.196-502, 1989. Disponível em: <http://web.utk.edu/ $\sim$ jmccart 1/McCarthy\%20and\%20ZacharaEST1989.pdf >. Acesso em: 03 mar. 2008.

MEYLAN, S. et al. Influence of metal speciation on natural freshwater on bioaccumulation of copper and zinc in periphyton: a microcosm study. Environmental Science and Technology, v.38, p.3104-3111, 2004. Disponível em:<http:/ /pubs.acs.org/doi/pdfplus/10.1021/es034993n. Acesso em: 20 mar. 2007. doi: 10.1021/es034993n.

MEYLAN, S. et al. Accumulation of copper and zinc in periphyton in response to dynamic variations of metal speciation in freshwater. Environmental Science and Technology, v.37, p.5204-5212, 2003. Disponível em:<http://pubs.acs.org/ doi/pdfplus/10.1021/es034566\%2B>. Acesso em: 20 mar. 2007. doi: $10.1021 /$ es $034566+$

MURPHY, J.; RILEY, J.P. A modified single solution method for the determination of phosphate in natural waters. Analytica Chimica Acta, v.27, p.31-36, 1962.

SILVA, L.S. Dinâmica da matéria orgânica e a interação com componentes inorgânicos na planta e no solo. 2001. 167f. Tese (Doutorado em Ciência do Solo) - Universidade Federal do Rio Grande do Sul. Faculdade de Agronomia, Porto Alegre, RS.

SMITH, D.S. et al. Metal speciation in natural waters with emphasis on reduced sulfur groups as strong metal binding sites. Comparative Biochemistry and Physiology, v.133, p.6574, 2002. Disponível em: <http://www.sciencedirect.com/ science $?$ ob $=$ MImg\&_imagekey=B 6W.89-46HB H4F-6H\&cdi $=6649 \&$ user $=687358 \&$ pii $=S 1532045602001084 \&$ orig=search\&cover Date $=09 \% 2 \mathrm{~F} 30 \% 2 \mathrm{~F} 2002 \& \mathrm{sk}=998669998 \& \mathrm{view}=\mathrm{c} \& w \mathrm{w} p=\mathrm{dGLbVlz}-$ zSkzS\&md5=2b36c 1 fee60ba987ce285e15a1d7343d\&ie=/ sdarticle.pdf >. Acesso em: 20 mar. 2007. doi:10.1016/S15320456(02)00108-4.

TEDESCO, M.J. et al. Análises de solo, planta e outros materiais. 2.ed. Porto Alegre: Universidade Federal do Rio Grande do Sul, 1995. 174p. (Boletim técnico, 5).

ZAMBROSI, F.C.B. Calagem e gessagem na especiação iônica da solução de um Latossolo sob sistema de plantio direto. 2004. 111f. Dissertação (Mestrado em Agronomia) Escola Superior de Agricultura Luiz Queiroz, Piracicaba, SP. 